\title{
Pgrmc1: new roles in the microglial mediation of progesterone-antagonism of estradiol-dependent neurite sprouting and in microglial activation
}

\author{
N. Bali ${ }^{1,2}$, T. E. Morgan ${ }^{1,2}$ and C. E. Finch ${ }^{1,2 *}$ \\ 1 Davis School of Gerontology, University of Southern California Los Angeles, Los Angeles, CA, USA \\ ${ }^{2}$ Dornsife College of Letters, Arts and Sciences, University of Southern California Los Angeles, Los Angeles, CA, USA
}

Edited by:

Sandra L. Petersen, University of

Massachusetts Amherst, USA

Reviewed by:

Sarah J. Spencer, Monash

University, Australia

Valerio Magnaghi, Università Degli

Studi di Milano, Italy

*Correspondence:

C. E. Finch, Davis School of

Gerontology and Dornsife College of

Letters, Arts and Sciences,

University of Southern California,

3715 McClintock Ave., Los Angeles,

CA 90089-0191, USA

e-mail: cefinch@usc.edu
Pgrmc1 (progesterone receptor membrane component 1) is a multifunctional $22 \mathrm{kDa}$ protein with heme-binding and P450-activating capacity which was recognized under different names for roles in cell motility during neural development and in cancer, and apoptosis. Pgrmc1 expression in microglia was recently shown by the present authors to mediate estrogen-progesterone interactions during axonal sprouting and to mediate microglial activation itself. We also discuss other functions of Pgramc1 in the nervous system and its possible relationship to the $18 \mathrm{kDa}$ sigma-2 receptor (S2R).

Keywords: Prgmc1, microglia, progesterone, sprouting, S2R

\section{INTRODUCTION}

Progesterone receptor membrane component 1 (Pgrmc1) is recognized for roles in many organs from independent discoveries; in neuroscience, its names include 25-Dx and VEMA (Cahill, 2007; Kimura et al., 2012). Other family members include Pgrmc2, neudesin, and neuferricin, which are distinct from the classical progesterone receptor (PR) transcription factors and from the large family of progestin/adipoQ membrane receptors that include $\mathrm{mPR} \alpha, \mathrm{mPR} \beta$, and $\mathrm{mPR} \gamma$. We recently discovered two new roles of Pgrmcl in microglial activation and in the microglial mediation of ovarian steroid effects on neurite sprouting (Bali et al., 2013).

Interactions of estradiol (E2) and progesterone (P4) are fundamental to reproductive cycles. In the uterus during the ovarian follicular phase, endometrial tissue growth is stimulated by plasma E2 elevations, while in the ovary, P4 regulates apoptosis of follicular granulosa cells via Pgrmc1 (Peluso et al., 2010; Peluso, 2013). During the ovarian luteal phase, if fertilized ova did not implant, plasma P4 levels fall, causing regression of endometrial tissues. A parallel process occurs in synapses of some neuronal systems. In the rat hippocampus, E2-driven dendritic spine numbers on CA1 pyramidal neurons increase during the follicular phase, followed by spine loss during the luteal phase $\mathrm{P} 4$ surge (Woolley and McEwen, 1992). Unlike the uterus and ovary, hippocampal synaptic cycles do not include neuron cell death.

E2-P4 interactions also modulate axonal regeneration during compensatory neuronal sprouting of the perforant path projections from the entorhinal cortex to the dentate gyrus molecular layer of the hippocampus. In ovariectomized (OVX) rats given entorhinal cortex lesions (ECL) to axotomize the perforant path, we found that P4 antagonized E2-dependent axonal outgrowth into the dentate gyrus molecular layer (Wong et al., 2009). Glial activation in the DG molecular layer peaks at days 3-4 post-ECL. By immunocytochemistry E2 implants decreased astrocyte (glial fibrillary acidic protein, GFAP) and microglial (isolectin B4) activation, whereas P4 antagonized E2-mediated decrease in glial activation, similar to its effects on neurite sprouting. This model may be used to optimize hormone therapy for axonal maintenance during the perforant path degeneration of early Alzheimer disease (Braak et al., 2006) and for neuroprotective effects of P4 in traumatic brain injury (TBI) (Stein, 2011).

These complex neuron-glial interactions were analyzed further with the "wounding-in-a-dish" model, in which embryonic rat E18 cortical neurons are grown on confluent glia (Wong et al., 2009). Axotomy by scratch-wounding induces neurite outgrowth, which was enhanced by $\mathrm{E} 2$ and antagonized by $\mathrm{E} 2+\mathrm{P} 4$ as in vivo with ECL. To our surprise, the E2-P4 antagonism of neurite outgrowth occurred with mixed glia (astrocytes:microglia, 3:1), but not with enriched astrocytes ( $>95 \%)$. This finding was unexpected because microglia reportedly lacked expression of Pgr, the classical PR (Sierra et al., 2008). Yet, P4 antagonism of E2dependent sprouting was blocked in mixed glia by two classical PR antagonists (ORG-31710 and RU-486) (Wong et al., 2009). Analysis of PRs of both astrocytes and microglia revealed new roles of Pgrmc1.

\section{PROGESTERONE RECEPTORS IN BRAIN CELLS}

Brain P4 actions are mediated by at least eight different proteins with differential expression in neurons and glia (Brinton et al., 2008). The two classic nuclear gene transcription factors PR-A and PR-B are alternate transcripts of $P g r$, are widely expressed in neurons throughout the adult rat brain including cerebral cortex, hippocampus, and hypothalamus (Intlekofer and Petersen, 2011; Bali et al., 2012). We detected PRs in astrocytes, but not 
in microglia of adult rat hippocampus (in situ hybridization and immunocytochemistry), and in primary glial cultures from neonatal cortex by rtPCR and Western blots (Figure 1) (Bali et al., 2013). These findings confirm observations of the absence of Pgr in ex vivo fluorescence-sorted (FACS) adult mouse brain microglia (Sierra et al., 2008). Pgr expression in astrocytes is also shown from antagonism of the P4:E2 interactions in neurite sprouting by ORG-31710 and RU-486, classical PR antagonists (Wong et al., 2009).

Pgrmcl was also found in microglia, astrocytes, and neurons, both in vivo and in vitro by immunocytochemistry, rtPCR, and Western blots (Figure 1). Unlike Pgr, Pgrmcl is uniformly
A

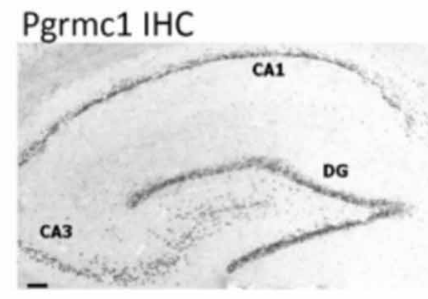

B

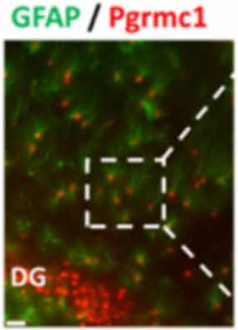

C IBA1/Pgrmc1

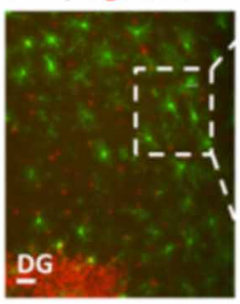

D GFAP / Pgrmc1

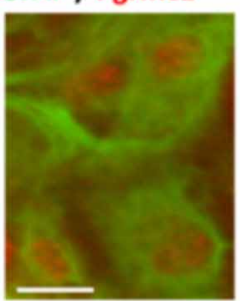

E

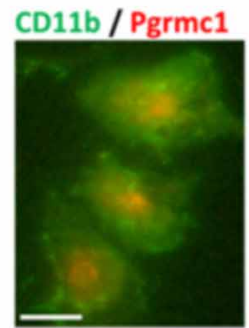

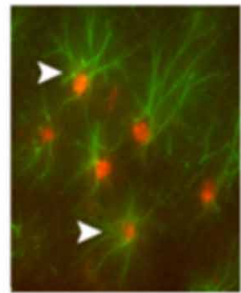

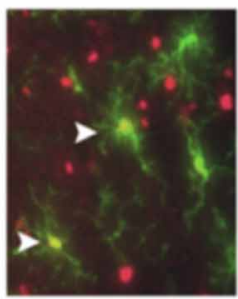

\section{GFAP}

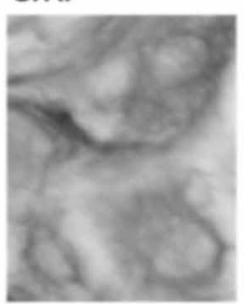

CD11b

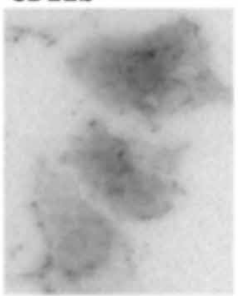

Pgr IHC

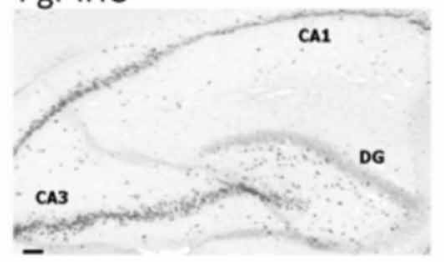

GFAP

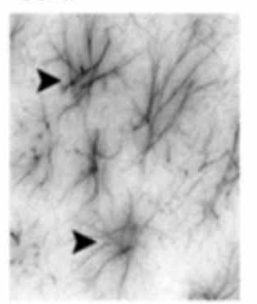

IBA1

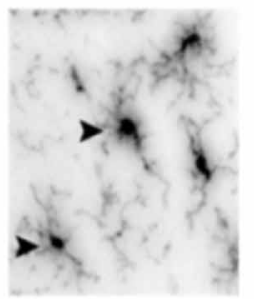

Pgrmc1

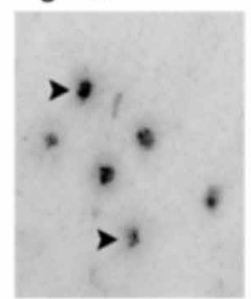

Pgrmc1

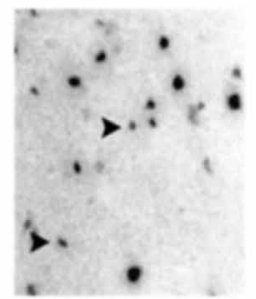

Pgrmc1

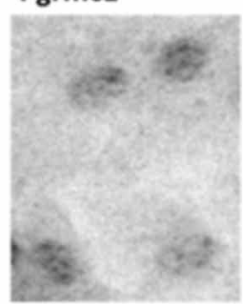

Pgrmc1

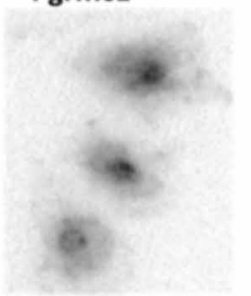

FIGURE 1 | Pgrmc1 and Pgr (PR) expression in rat brain glia and neurons by immunocytochemistry. (A) Neuronal Pgrmc1 and Pgr expression in adult rat hippocampus was equivalent in the hippocampus CA1, CA3, and dentate gyrus (DG) layers. In contrast, neuronal Pgr expression was lower DG vs. CA1 and CA3 layers. (B) Astrocyte Pgrmc1 in DG molecular layer co-stained with GFAP.
Arrowheads point to co-labeled astrocytes. (C) Microglial Pgrmc1 in DG molecular layer co-stained with IBA1. Arrowheads point to co-labeled microglia. (D) Astrocyte Pgrmc1 in primary cultures from cerebral cortex co-stained with GFAP. (E) Microglial Pgrmc1 in primary cultures co-stained with CD11b. Scale bars, $20 \mu \mathrm{m}$. Modified from Bali et al. (2012) and Bali et al. (2013). 
expressed by all hippocampal neurons (Bali et al., 2012). Although initially characterized as a membrane receptor, Pgrmc1 can reside in the cell nucleus (Peluso et al., 2010; Bali et al., 2013). We also detected other membrane-associated PRs, $\operatorname{mPR} \alpha, \operatorname{mPR} \beta$, and $\mathrm{mPR} \gamma$, in astrocytes and microglia (rtPCR, DNA sequence confirmed; unpublished data). Meffre et al. (2013) also reported $\mathrm{mPR} \alpha$ in astrocytes and microglia, but only after TBI. In spinal cord, $\mathrm{mPR} \alpha$ was found in glia, but not $\mathrm{mPR} \beta$ (Labombarda et al., 2010). We conclude that P4 actions in microglia are mediated by membrane PRs because they lack expression of the classical PRs. A specific role of Pgrmcl in microglial activation is described below.

The neuronal side of Pgrmcl began in 1999, under the name of VEMA, a protein that proved to regulate neuron outgrowth in mouse and nematode (Runko et al., 1999; Cahill, 2007). Its first brain steroidal association was " $25-\mathrm{Dx}$," from a cDNA encoding a $25-\mathrm{kDa}$ protein that responded to E2 and P4 (Krebs et al., 2000). In hypothalamic neurons, 25-Dx induction by E2 was blocked by P4. However, in hippocampal neurons of OVX rats, P4 did not antagonize E2-induced Pgrmcl expression; moreover, P4 was as strong an inducer of Pgrmc1 as E2 alone (Bali et al., 2012). Its hypothesized role in reproductive functions was shown in GnRH neurons, where the inhibition of $\left[\mathrm{Ca}^{+2}\right]_{i}$ oscillations by $\mathrm{P} 4$ was blocked by AG-205, a Pgrmcl ligand inhibitor, but not by RU486, the classic PR antagonist (Bashour and Wray, 2012). In neural progenitor cells, P4 was pro-proliferative via Pgrmcl (Liu et al., 2009). On the glial side, Pgrmcl was detected in both astrocytes and microglia, noted above. TBI also induced Pgrmcl in adjacent astrocytes and neurons (Meffre et al., 2005).

\section{Pgrmc1 AND THE ROLE OF MICROGLIAL ACTIVATION IN P4-E2 ANTAGONISM}

As noted above, P4 antagonized E2-induced neurite sprouting in mixed glia containing 3:1 astrocytes:microglia, but the antagonism was absent from enriched astrocytes from

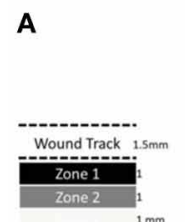

CD11b
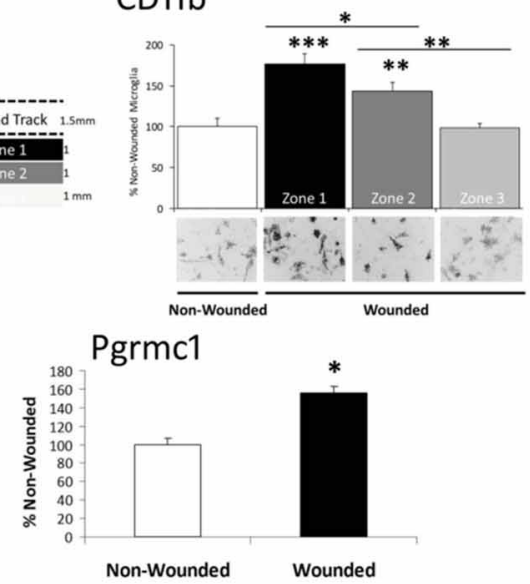

C

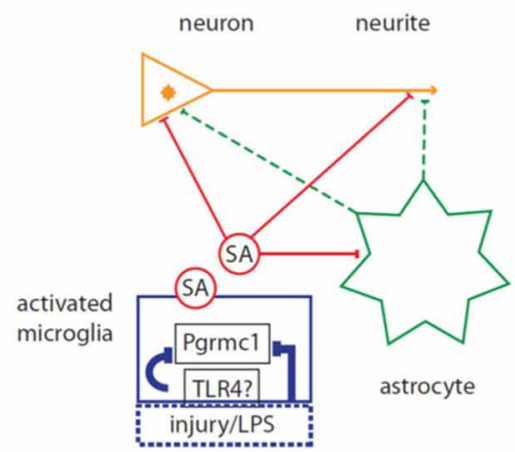

FIGURE 2 | Microglial activation via scratch wounding and LPS. (A) Microglial CD11b in 3 zones (1 mm wide) progressively decreased distal to the wound. Pgrmc1 protein was also induced by scratch wounding. ${ }^{* * *} p<0.0001 ;{ }^{* *} p<0.03 ;{ }^{*} p<0.05$ vs non-wounded. (B) CD11b and Pgrmc1 protein were both induced by by LPS. ${ }^{* * *} p<0.0001$; ${ }^{* *} p<0.001$ vs vehicle. (C) Model to show targets of the undefined soluble activity (SA) from activated microglia and microglial Pgrmc1 that mediate the antagonism of P4 on E2-dependent neurite outgrowth. Microglial activation
B
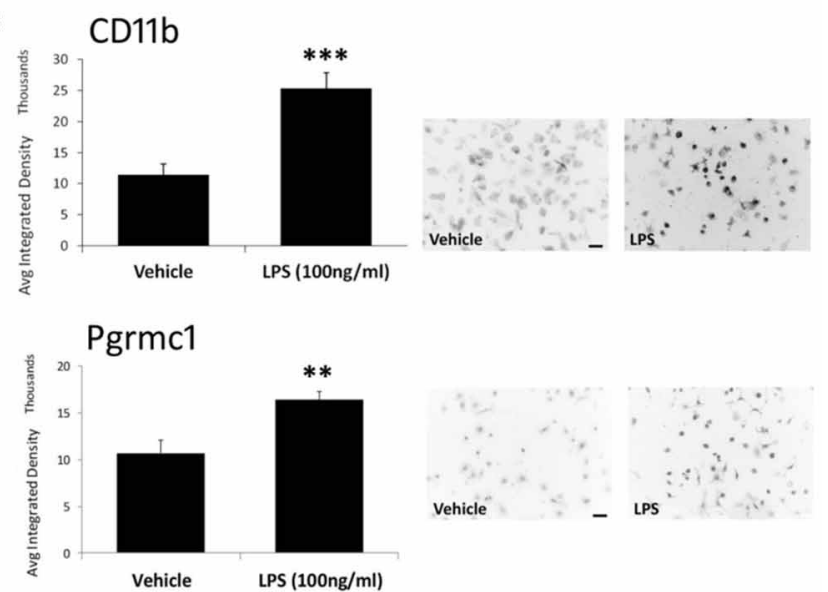

by LPS is assumed to be mediated by toll-like receptor-4 (TLR4); activation by scratch wounding has undefined pathways. The schema shows two possible SA actions on neurites: direct effects (solid red lines) on neurite outgrowth at the neurite growth cone (arrowhead) and/or involving the neuronal nucleus; indirect effects (dashed green lines) via astrocyte secretions on neurite or neuronal nucleus. Glial activation by injury or LPS and the SA effects were blocked by Pgrmc1 knockdown. Modified from Bali et al. (2013). 
which microglia had been removed (>95\% astrocytes) (Wong et al., 2009). A caveat is that the $4 \mathrm{~h}$ mechanical shaking process to remove microglia could alter glial responses, e.g., hydrodynamic forces induce astrocyte aromatase (Gatson et al., 2011). The role of microglia was shown in a new protocol to add-back microglia, which restored the P4-E2 antagonism of neurite outgrowth (Bali et al., 2013).

The add-back protocol also allowed us to separately manipulate PRs in astrocytes and microglia before the mixed glia reconstitution. An astrocyte role was anticipated by the Pgrmcl dependence of BDNF secretion ( $\mathrm{Su}$ et al., 2012). Using siRNA, we showed that Pgrmcl knockdown in microglia, but not in astrocytes abolished the P4-E2 antagonism of neurite outgrowth. We then found that conditioned media from scratch-wounded cultures of microglia alone sufficed to restore the P4-E2 antagonism. To identify the simplest conditions that restored P4-E2 antagonism, we used charcoal-stripped FBS containing minimal steroids. However, the soluble activity was absent from unwounded microglia, implying a role of microglial activation. The requirement for microglial activation also explained why $\mathrm{P} 4$ did not antagonize E2-dependent outgrowth in non-wounded cultures. A candidate for the soluble factor was $\mathrm{TNF} \alpha$, which is released by microglia and which can inhibit neurite outgrowth; however TNF $\alpha$ levels did not differ with scratch wounding in our model (unpublished).

Because scratch-wounding is an unconventional mode of microglial activation (we did not find other reports), we examined the classical microglial activator, LPS, which restored the soluble activity. Pgrmcl protein was also induced in microglia by scratch-wounding or by LPS (Figures 2A,B), consistent with its induction in astrocytes by TBI, noted above. Moreover, Pgrmcl knockdown by siRNA abolished LPS microglial activation by the standard CD11b marker. The expression of Pgrmc1 in DG microglia (Figure 1E) could thus mediate in vivo effects of $\mathrm{P} 4$ on neurite outgrowth.

Thus, Pgrmc1 is not only a new marker of microglial activation, but has a fundamental role in microglial activation itself. Therefore, Pgrmcl may regulate microglial-mediated neurotoxic, and perhaps, neuroprotective activities, as well as synaptic pruning (Aguzzi et al., 2013). The responses to LPS imply a link to toll-like receptor pathways, e.g., TLR4, which mediates microglial activation by LPS (Chen et al., 2012) (Figure 2C). Its role in the activation of other monocytes is unknown. There may also be interactions of P4 and its metabolites with other receptors throughout the body, e.g., GABA receptors.

\section{REFERENCES}

Aguzzi, A., Barres, B. A., and Bennett, M. L. (2013). Microglia: scapegoat, saboteur, or something else? Science 339, 156-161. doi: 10.1126/science. 1227901

Ahmed, I. S., Rohe, H. J., Twist, K. E., Mattingly, M. N., and

\section{POSSIBLE RELATIONSHIP OF Pgrmc1 TO THE SIGMA-2 RECEPTOR (S2R)}

The S2R was reportedly identified as Pgrmcl and 25-Dx by mass spectrometry on the basis of 29 shared residues (Xu et al., 2011). Both are also heme binding proteins with high affinity for P4 (Cahill, 2007; Johannessen et al., 2011; Peluso, 2013). The S2R is of increasing interest in various research areas which do not consistently cross-reference Pgrmc1, including apoptosis, cancer metastasis, cocaine addiction, and ion channels. Of relevance to our findings, S2R mediates microglial migration up gradients of ATP in vitro, with pharmacological specificities that appear distinct from P4-like ligands (Cuevas et al., 2011). However, its $18 \mathrm{kDa}$ size is distinctly smaller than $25 \mathrm{kDa}$ Pgrmcl (Ruoho et al., 2013), and could be an alternatively spliced form.

Whatever the case, S2R and Pgrmc1 both mediate cell migration in diverse contexts. Recall that Pgrmcl/VEMA influenced axonal outgrowth in mice and nematodes at developmental stages (Runko et al., 1999) which are not known for P4-dependence. The activation of microglia and induction of Pgrmcl in vitro in the absence of exogenous P4 (see above) may be another P4independent effect (Mir et al., 2012). However, local P4 could have a role via the cytochrome b5-like heme/steroid-binding function of Pgrmcl which interacts with diverse binding partners, including P450 enzymes (Cahill, 2007; Ahmed et al., 2010; Kimura et al., 2012).

\section{CONCLUSIONS AND OPEN OUESTIONS}

The diverse functions of Pgrmcl continue to expand in many fields that have little cross-talk. Further progress may depend as much on biochemistry as further elegant molecular genetics. Pgrmcl knockout mice may become available. The connections with innate immunity through the toll-like receptors are an attractive target. The identification of proteins or other molecules in the soluble activity from conditioned media could be done efficiently. The requirement for microglial activation in the P4-E2 antagonism of neurite outgrowth further suggests that microglial activation, such as arises during aging and Alzheimer disease, might alter the absence of P4-E2 antagonism in regulation of hippocampal neuron Pgrmcl in vivo (Bali et al., 2012). Given the role of Pgrmcl in these experiments and the finding that Pgrmcl expression influences astrocyte BDNF (Su et al., 2012), we anticipate further roles of Pgrmcl in the complex biology of glia-neuron interactions.

\section{ACKNOWLEDGMENTS}

This work was supported by NIH grant 2P01 AG026572-06 to Roberta D. Brinton; Project 2 to C. E. Finch and T. E. Morgan; Animal Core to T. E. Morgan. We also thank John Peluso, Javier Cuevas, and Arnold E. Ruoho for helpful comments.

Bali, N., Arimoto, J. M., Iwata, N., Lin, S. W., Zhao, L., Brinton, R. D., et al. (2012). Differential responses of progesterone receptor membrane component-1 (Pgrmcl) and the classical progesterone receptor (Pgr) to 17beta-estradiol and progesterone in hippocampal subregions that support synaptic remodeling and neurogenesis. Endocrinology 153, 759-769. doi: 10.1210/en.2011-1699

Bali, N., Arimoto, J. M., Morgan, T. E., and Finch, C. E. (2013). Progesterone antagonism of neurite outgrowth depends on microglial 
activation via Pgrmcl/S2R. Endocrinology 154, 2468-2480. doi: 10.1210/en.2012-2109

Bashour, N. M., and Wray, S. (2012). Progesterone directly and rapidly inhibits GnRH neuronal activity via progesterone receptor membrane component 1. Endocrinology 153, 4457-4469. doi: 10.1210/en.20121122

Braak, H., Alafuzoff, I., Arzberger, T., Kretzschmar, H., and Del Tredici, K. (2006). Staging of Alzheimer disease-associated neurofibrillary pathology using paraffin sections and immunocytochemistry. Acta Neuropathol. 112, 389-404. doi: 10.1007/s00401-006-0127-z

Brinton, R. D., Thompson, R. F., Foy, M. R., Baudry, M., Wang, J., Finch, C. E., et al. (2008). Progesterone receptors: form and function in brain. Front. Neuroendocrinol. 29:313-339. doi: 10.1016/j.yfrne.2008.02.001

Cahill, M. A. (2007). Progesterone receptor membrane component 1 : an integrative review. J. Steroid Biochem. Mol. Biol. 105, 16-36. doi: 10.1016/j.jsbmb.2007.02.002

Chen, Z., Jalabi, W., Shpargel, K. B., Farabaugh, K. T., Dutta, R., Yin, X., et al. (2012). Lipopolysaccharideinduced microglial activation and neuroprotection against experimental brain injury is independent of hematogenous TLR4. J. Neurosci. 32, 11706-11715. doi: 10.1523/JNEUROSCI.0730-12.2012

Cuevas, J., Rodriguez, A., Behensky, A., and Katnik, C. (2011). Afobazole modulates microglial function via activation of both sigma-1 and sigma-2 receptors. J. Pharmacol. Exp. Ther. 339, 161-172. doi: 10.1124/jpet.111.182816

Gatson, J. W., Simpkins, J. W., Yi, K. D., Idris, A. H., Minei, J. P., and Wigginton, J. G. (2011). Aromatase is increased in astrocytes in the presence of elevated pressure. Endocrinology 152, 207-213. doi: 10.1210/en.2010-0724

Intlekofer, K. A., and Petersen, S. L. (2011). Distribution of mRNAs encoding classical progestin receptor, progesterone membrane components 1 and 2, serpine mRNA binding protein 1 , and progestin and ADIPOQ receptor family members 7 and 8 in rat forebrain. Neuroscience 172, 55-65. doi: 10.1016/j.neuroscience.2010.10.051 Johannessen, M., Fontanilla, D., Mavlyutov, T., Ruoho, A. E., and Jackson, M. B. (2011). Antagonist action of progesterone at sigma-receptors in the modulation of voltage-gated sodium channels. Am. J. Physiol. Cell Physiol. 300, C328-C337. doi: 10.1152/ajpcell.00383.2010

Kimura, I., Nakayama, Y., Konishi, M., Terasawa, K., Ohta, M., Itoh, N., et al. (2012). Functions of MAPR (membrane-associated progesterone receptor) family members as heme/steroid-binding proteins. Curr. Protein Pept. Sci. 13, 687-696.

Krebs, C. J., Jarvis, E. D., Chan, J., Lydon, J. P., Ogawa, S., and Pfaff, D. W. (2000). A membrane-associated progesterone-binding protein, 25Dx, is regulated by progesterone in brain regions involved in female reproductive behaviors. Proc. Natl. Acad. Sci. U.S.A. 97, 12816-12821. doi: 10.1073/pnas.97.23.12816

Labombarda, F., Meffre, D. Delespierre, B., KrivokapicBlondiaux, S., Chastre, A., Thomas, P., et al. (2010). Membrane progesterone receptors localization in the mouse spinal cord. Neuroscience 166, 94-106. doi: 10.1016/j.neuroscience.2009.12.012

Liu, L., Wang, J., Zhao, L., Nilsen, J., McClure, K., Wong, K., et al. (2009). Progesterone increases rat neural progenitor cell cycle gene expression and proliferation via extracellularly regulated kinase and progesterone receptor membrane components 1 and 2. Endocrinology 150, 3186-3196. doi: 10.1210/en.20081447

Meffre, D., Delespierre, B., Gouezou, M., Leclerc, P., Vinson, G. P., Schumacher, M., et al. (2005). The membrane-associated progesterone-binding protein 25Dx is expressed in brain regions involved in water homeostasis and is up-regulated after traumatic brain injury. J. Neurochem. 93, 1314-1326. doi: 10.1111/ j.1471-4159.2005.03127.x
Meffre, D., Labombarda, F., Delespierre, B., Chastre, A. De Nicola, A. F., Stein, D. G. et al. (2013). Distribution of membrane progesterone receptor alpha in the male mouse and rat brain and its regulation after traumatic brain injury. Neuroscience 231, 111-124. doi: 10.1016/j.neuroscience.2012.11.039

Mir, S. U., Ahmed, I. S., Arnold, S., and Craven, R. J. (2012). Elevated progesterone receptor membrane component $1 /$ sigma- 2 receptor levels in lung tumors and plasma from lung cancer patients. Int. J. Cancer 131, E1-E9. doi: 10.1002/ijc. 26432

Peluso, J. J. (2013). Progesterone receptor membran component 1 and its role in ovarian follicular growth. Front. Neurosci. 7:99. doi: 10.3389/fnins.2013.00099

Peluso, J. J., Liu, X., Gawkowska, A., Lodde, V., and $\mathrm{Wu}, \mathrm{C} . \mathrm{A}$ (2010). Progesterone inhibits apoptosis in part by PGRMC1regulated gene expression. Mol. Cell. Endocrinol. 320, 153-161. doi: 10.1016/j.mce.2010.02.005

Ruoho, A. E., Guo, L. W., Hajipour, A. R., Karaoglu, K., Mavlyutov, T. A., Chu, U. B., et al. (2013). Will the true Sigma-2 receptor please stand up. Soc. Neurosci. Abstr. 631.08

Runko, E., Wideman, C., and Kaprielian, Z. (1999). Cloning and expression of VEMA: a novel ventral midline antigen in the rat CNS. Mol. Cell. Neurosci. 14, 428-443. doi: 10.1006/mcne.1999.0794

Sierra, A., Gottfried-Blackmore, A., Milner, T. A., McEwen, B. S., and Bulloch, K. (2008). Steroid hormone receptor expression and function in microglia. Glia 56, 659-674 doi: 10.1002/glia.20644

Stein, D. G. (2011). Progesterone in the treatment of acute traumatic brain injury: a clinical perspective and update. Neuroscience 191, 101-106. doi 10.1016/j.neuroscience.2011.04.013

$\mathrm{Su}, \quad$ C., Cunningham, R. L., Rybalchenko, N., and Singh, M. (2012). Progesterone increases the release of brain-derived neurotrophic factor from glia via progesterone receptor membrane component 1 (Pgrmc1)dependent ERK5 signaling. Endocrinology 153, 4389-4400. doi: 10.1210/en.2011-2177

Wong, A. M., Rozovsky, I., Arimoto, J. M., Du, Y., Wei, M., Morgan, T. E., et al. (2009). Progesterone influence on neurite outgrowth involves microglia. Endocrinology 150, 324-332. doi: 10.1210/en.2008-0988

Woolley, C. S., and McEwen, B. S. (1992). Estradiol mediates fluctuation in hippocampal synapse density during the estrous cycle in the adult rat. J. Neurosci. 12, 2549-2554.

Xu, J., Zeng, C., Chu, W., Pan, F., Rothfuss, J. M., Zhang, F., et al. (2011). Identification of the PGRMC1 protein complex as the putative sigma-2 receptor binding site. Nat. Commun. 2, 380. doi: 10.1038/ncomms1386

Conflict of Interest Statement: The authors declare that the research was conducted in the absence of any commercial or financial relationships that could be construed as a potential conflict of interest.

Received: 13 July 2013; accepted: 13 August 2013; published online: 03 September 2013.

Citation: Bali N, Morgan TE and Finch CE (2013) Pgrmcl: new roles in the microglial mediation of progesteroneantagonism of estradiol-dependent neurite sprouting and in microglial activation. Front. Neurosci. 7:157. doi: 10.3389/fnins.2013.00157

This article was submitted to Neuroendocrine Science, a section of the journal Frontiers in Neuroscience. Copyright (c) 2013 Bali, Morgan and Finch. This is an open-access article distributed under the terms of the Creative Commons Attribution License (CC BY). The use, distribution or reproduction in other forums is permitted, provided the original author(s) or licensor are credited and that the original publication in this journal is cited, in accordance with accepted academic practice. No use, distribution or reproduction is permitted which does not comply with these terms. 